\title{
Compact non-left-recursive grammars using the selective left-corner transform and factoring*
}

\author{
Mark Johnson Brian Roark \\ Cognitive and Linguistic Scionces, Box 1978 \\ Brown University \\ Mark Johnson@Brown.edu Brian_Roark@Brown.edu
}

\begin{abstract}
The left-corner transform removes left-recursion from (probabilistic) context-free grammars and unification grammass, permitting simple top-down parsing techniques to bo used. Unfortunately tho grammans produced by the standand left-comer transform are usually much larger than the original. The selective left-corner transfom described in this paper produces a transformed grammale which simulates left-comer recognition of a nser-specified set of the original productions, and top-down recognition of the othors. Combined with two factorizations, it produces non-left-recursive grammans that aro not much larger than the original.
\end{abstract}

\section{Introduction}

Top-down parsing techniques are at,tactive because of their simplicity, and can ofton achieve good porformance in practice (Roark and .Johnson, 1999). However, with a left-recursive grammar such parsers typically fail to terminate. 'J'he left-comer granmar transform converts a left-recursive grammant into a non-left-recursive one: a top-down parser using' a left-cornes transformed grammar sinulates a left-corner parser using the original grammar. (Rosenkrantz and Lewis II, 1970; Aho and Ullman, 1972). However, the left-corner transformed grammax can be significantly larger than the original grammar, causing numcrous problenss. For example, we show bolow that a probabilistic context-free grammar (PCFG) estimated from left-corner transformed Penn WSJ trec-bank trees exhibits considcrably greater sparse data problems than a PCFG estimated in the usual manmer, simply because the left-corner transformed grammar contains approximately 20 times more productions. The transform described in this paper produces a grammar approximately the same size as the input grammar, which is not as adversely affected by sparse data.

\footnotetext{
* 'This research was supported by NST' awards 9720368, 9870676 and 9812169 . Wo would like to thank oul colleagues in 13IJIP (Brown Labonatory for Linguistic Information Processing) and 3ot Moore for their helpful comments on this pajper.
}

Jeft-corner transforms are particularly useful becanse they can preserve ammotations on productions (more on this bolow) and are therefore applicable to more complex grammar formalisms as woll as CFGs; a property which other approaches to left-recursion climination typically lack. For example, they apply to left-recursive unification-based grammars (Matsumoto et al., 1983; Pereira and Shicber, 1987; Johnsom, 1998a). Bocause the emission probability of a PCTG production can be regarded as an amotation on a CFG production, the left-comor transform can produce a CFG with weighted productions which assigns the same probabilitios to strings and transformed trees as the original grammar (Abney et al., 1999). However, the transformed grammars can bo much larger than the original, which is unacceptablo for many applications involving largo grammars.

The selective left-coner transform reduces the transformed grammar size because only those productions which appear in a left-recursive cycte necd be recognized left-comes in order to remove leftrecursion. A top-down parser using a grammar produced by the selective left-corner transform simulates a generalized lefl-corner parser (Doners, 1977 Nijholt, 1980) which recognizes a user-specified subset of the original productions in a left-corner fashion, and the other productions top-down.

Although wo do not investigate it in this paper, the selective left-corner transform should ustally have a smaller search space relative to the standard left-corner transform, all else being equal. The partial parses produced during a top-down parse consist of a single connected tree fragment, while the partial parses produced produced during a left-corner parse generally consist of several discomected treo fragments. Since these fragmonts are only wealkly related (via the "link" constraint described bolow), the search for each fragment is rolatively independent. This may be responsible for the obscrvation that, exhaustive left-corner parsing is less efficient than t,op)-clown parsing (Covington, 1994). Informally, because the selective left-corner transform recognizes only a subset of productions in a left-corner fashion, its partial parses contain fewer tree discontiguous 
fragments and the search may be more efficient.

While this paper focuses on reducing grammar size to minimize sparse data problems in PCFG estimation, the modified left-corner transforms described here are generally applicable wherever the original left-corner transform is. For cxample, the selective left-corner transform can be used in place of the standard left-corner transform in the construction of finite-state approximations (Johnson, 1998a), often reducing the size of the intermediate automata constructed. The selective left-corner transform can be generalized to head-corner parsing (van Noord, 1997), yielding a selective head-cornor parser. (This follows from generalizing the selective left-corner transform to Horn clauses).

After this paper was accepted for publication we learnt of Moore (2000), which addresses the issue of grammar size using very similar techniques to those proposed here. The goals of the two papers are slightly different: Moore's approach is designed to reduce the total grammar size (i.e., the sum of the lengths of the productions), while our approach minimizes the number of productions. Moore (2000) does not address left-corner tree-transforms, or questions of sparse datia and parsing accuracy that are covered in section 3 .

\section{The selective left-corner and related transforms}

This section introduces the selective left-corner transform and two additional factorization transforms which apply to its output. These transforms are used in the experiments described in the following section. As Moore (2000) observes, in general the transforms produce a non-left-1ecursive output grammar only if the input grammar $G$ does not contain unary cycles, i.e., there is no nonterminal $A$ such that $A \rightarrow_{G}^{+} A$.

\subsection{The selective left-corner transform}

The selective left-corner transform takes as input a CFG $G=(V, T, P, S)$ and a set of left-corner productions $L \subseteq P$, which contains no cpsilon productions; the non-left-corner productions $P-L$ are called topdown productions. The standard left-corner transform is obtaincd by setting $L$ to the set of all non-epsilon productions in $P$. The selective leftcorner transform of $G$ with respect to $L$ is the $C F G$ $\mathcal{L C}_{L}(G)=\left(V_{1}, T, P_{1}, S\right)$, where:

$$
V_{1}=V \cup\{D-X: D \in V, X \in V \cup T\}
$$

and $P_{1}$ contains all instances of the schemata 1 . In these schemata, $D \in V, w \in T$, and lower case greek letters range over $(V \cup T)^{*}$. The $D-X$ are new nonterminals; informally they cncode a parse state in which an $D$ is predicted top-down and an $X$

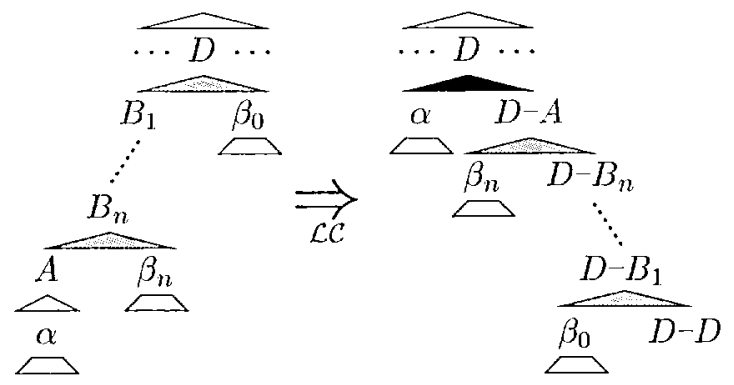

Figure 1: Schematic parse trees gencrated by the original grammar $G$ and the selective left-corner transformed grammar $\mathcal{L C}_{J}(G)$. The shaded local trees in the original parse tree correspond to left-corner productions; the corresponding local trees (generated by instances of schema 1c) in the selective left-corner transformed trec are also shown shaded. The local tree colored black is generated by an instance of sclicna $1 \mathrm{~b}$.

has been found left-corner, so $D-X \Rightarrow_{\mathcal{L} \mathcal{C}_{L}(G)}^{*} \gamma$ only if $D \Rightarrow_{G}^{*} X \gamma$.

$$
\begin{array}{ll}
D \rightarrow w D-w & \\
D \rightarrow \alpha D-A & \text { where } A \rightarrow \alpha \in P- \\
D-B \rightarrow \beta D-C & \text { where } C \rightarrow B \beta \in L \\
D-D \rightarrow C &
\end{array}
$$

The schemata function as follows. The productions introduced by schema la start a left-comer parse of a predicted nonterminal $D$ with its leftmost terminal $w$, while those introduced by schema $1 \mathrm{~b}$ start a left-corner parse of $D$ with a left-corncr $A$, which is itself found by the top-down recognition of production $A \rightarrow \alpha \in P-L$. Schema 1c cxtends the current left-corner $B$ up to a $C$ with the left-corner recognition of production $C \rightarrow B \beta$. Finally, schema 1d matches the top-down prediction with the recognized left-corner category.

Figure 1 schematically depicts the relationship between a chain of left-corner productions in a parse tree generated by $G$ and the chain of corresponding instances of schema 1c. The left-corner recognition of the chain starts with the recognition of $\alpha$, the right-hand side of a top-down production $A \rightarrow \alpha$, using an instance of schema $1 \mathrm{~b}$. The left-branching chain of left-corner productions corresponds to a right-branching chain of instances of schema $1 \mathrm{c}$; the left-corner transform in effect converts left recursion into right recursion. Notice that the top-down predicted category $D$ is passed down this right-recursive chain, effectively multiplying each left-corner productions by the possible top-down prodicted categorics. The right recursion terminates with an instance of schema 1d when the left-corner and topdown categories match.

Figure 2 shows how top-down productions from $G$ are recognized using $\mathcal{L C}_{I}(G)$. When the se- 


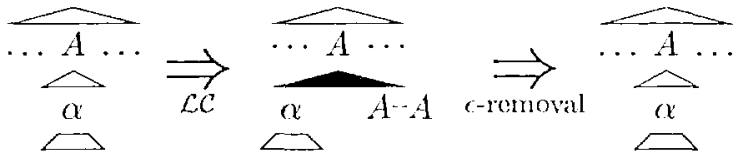

Figure 2: The recognition of a top-down procluction $A \rightarrow$ $\alpha$ by $\mathcal{L C}_{L}(G)$ involves a left-corner category $A-A$, which immediately rewrites to $\epsilon$. Onc-step $\epsilon$-removal applicel to $\mathcal{L C}_{L}(G)$ produces a grammar in which each top-down production $A \rightarrow \alpha$ corresponds to a production $A \rightarrow \alpha$ in the transformed grammar.

lective left-conner transform is followed by a oncstep c-removal transform (i.e., composition or partial cvaluation of schema $1 b$ with respect to schema 1d (Johnson, 1998a; Abncy and Johnson, 1991; Resnik, 1992)), cach top-down production from $G$ appears unchanged in the final grammar. Full e-romoval yichds the grammar given by the schematia below.

$$
\begin{array}{ll}
D \rightarrow w D-w & \\
D \rightarrow w & \text { where } D \Rightarrow+w \\
D \rightarrow \alpha D-A & \text { where } A \rightarrow \alpha \in P^{+}, L \\
D \rightarrow \alpha & \text { where } D \Rightarrow{ }_{L}^{*} A, A \rightarrow \alpha \in P-I \\
D-B \rightarrow \beta D C & \text { where } C \rightarrow B \beta \in I \\
D-B \rightarrow \beta & \text { where } D \Rightarrow_{L}^{*} C, C \rightarrow B \beta \in I
\end{array}
$$

Moore (2000) introduces a version of the leftcorner transform called $\mathrm{LC}_{L, R}$, which applies only to productions with left-recursive parent and left child categories. In the context of the other transforms that Moore introduces, it secms to have the samo oflect in his system as the selective left-comer transform docs hore.

\subsection{Selective left-corner tree transforms}

There is a 1-to-1 correspondence between the parse troes generated by $G$ and $\mathcal{L C} \mathcal{C}_{L}(G)$. A tree $t$ is generated by $G$ iff there is a corresponding $t^{\prime}$ generated by $\mathcal{L C}_{I}(G)$, where cach occurrence of a top-down production in the derivation of $t$ corresponds to cxactly one local tree generated by occurrence of the corresponding instance of schema $1 . b$ in the derivation of $t^{\prime}$, and each occurrence of a left-corner production in $t$ colresponds to exactly one occurrence of the corresponding instance of schema $1 \mathrm{c}$ in $t^{\prime}$. It is straightforward to define a 1-to-1 tree transform $\mathcal{T}_{L}$ mapping parse trecs of $G$ into parse trees of $\mathcal{C} C_{L_{L}}(G)$ (Johnson, 1998a; Roark and Johnson, 1999). In the empirical cvaluation below, we estimate a $\mathrm{PCFG}$ from the trees obtained by applying $\mathcal{T}_{J}$, to the trees in the Penn WSJ tree-bank, and compare it to the PCFG estimatied from tho original tree-bank trees. A stochastic top-down parser using the PCFG estimated from the trees produced by $\mathcal{T}_{L}$, simulates a stochastic generalized left-corner parser, which is a gencralization of a standard stochastic left-corner parser that permits productions to be recognized top-down as well as left-corner (Mamning and Carpenter, 1997). Thus investigating the properties of PCTG estimated from trees transformed with $\mathcal{T}_{L}$ is an casy way of studying stochastic push-clown automatia performing generalized left-comer parses.

\subsection{Pruning useless productions}

We turn now to the problem of reducing the size of the grammars produced by left-corner transforms. Many of the productions generated by schemata 1 are useless, i.c., they never appear in any terminating derivation. While they can be removed by standard methods for deleting useless productions (IIopcroft and Ullman, 1979), the relationship between the parse trees of $G$ and $\mathcal{L C} L(G)$ depicted in Figure 1 shows low to determine ahead of time the now nonterminals $D-X$ that can appear in useful productions of $\mathcal{L C} C_{L}(G)$. This is known as a link constraint.

For $(P) C I G$ s there is a particularly simple link constraint: $J)-X$ appears in useful productions of $\mathcal{L C} C_{L}(G)$ only if $\exists \gamma \in(V \cup T)^{\star} . D \Rightarrow_{L}^{\star} X \gamma$. If epsilon removal is applied to the resulting grammar, $D-X$ appears in uscful productions only if $\exists \gamma \in(V \cup T)^{+} . D \Rightarrow_{L_{2}}^{\star} X \gamma$. Thus one only need generate instances of the left-corner schemata which satisfy the corresponding link constraints.

Moore (2000) suggests an additional constraint on nonteminals $D X$ that can appear in useful productions of $\mathcal{L C} C_{L}(G): D$ must cither be the start symbol of $G$ or else appear in a production $A \rightarrow \alpha D \beta$ of $G$, for any $A \in V, \alpha \in\{Y \cup T\}^{+}$and $\left.\beta \in\left\{Y \cup^{\prime}\right]^{\prime}\right\}^{*}$. It is easy to see that the productions that Moore's constraint prohibits are useless. There is one nontorminal in the tree-bank grammar investigated below that has this property, namely LST. However, in the tree-bank grammar none of the productions expanding LST are left-recursive (in fact, the first child is always a preterminal), so Moore's constraint does not affect the size of the transformed grammars investigated below.

While these constraints can dramatically reduce both the number of productions and the size of the parsing soarch space of the transformed grammar, in general the transformed grammar $\mathcal{L} \mathcal{C}_{J}(G)$ can be quadratically larger than $G$. There are two causes for the explosion in grammar size. First, $\mathcal{L C} C_{J_{L}}(G)$ contains an instance of schema $1 \mathrm{~b}$ for cach topdown production $A \rightarrow \alpha$ and each $D$ such that $\exists \gamma . D \Rightarrow_{L}^{*} A \gamma$. Sccond, $\mathcal{L C}_{I}(G)$ contains an instance of schema 1c for cach left-corner production $C \rightarrow \beta$ and each $D$ such that $\exists \gamma . D \Rightarrow_{L}^{\star} C \gamma$. In effect, $\mathcal{L C}_{L}(G)$ contains one cony of each production for cach possible left-corner ancestor. Section 2.5 describes further factorizations of the productions of $\mathcal{L C} C_{L}(G)$ which mitigate these causes. 


\subsection{Optimal choice of $L$}

Because $\Rightarrow_{L}^{\star}$ increases monotonically with $\Rightarrow_{L}$ and hence $L$, we typically reduce the size of $\mathcal{L C} C_{L}(G)$ by making the left-corner production set $L$ as small as possible. This section shows how to find the unique minimal set of left-corner productions $L$ such that $\mathcal{L C}_{L}(G)$ is not left-recursive.

Assume $G=(V, T, P, S)$ is pruned (i.e., $P$ contains no useless productions) and that there is no $A \in V$ such that $A \rightarrow_{G}^{+} A$ (i.e., $G$ does not generate recursive unary branching chains). For reasons of space we also assume that, $P$ contains no $\epsilon$-productions, but this approach can be extended to deal with them if desired. A production $A \rightarrow B \beta \in$ $P$ is left-recursive iff $\exists \gamma \in(V \cup T)^{\star} . B \Rightarrow_{l}^{*} A \gamma$, i.e., $P$ rewrites $B$ into a string beginning with $A$. Let $L_{0}$ be the set of left-recursive productions in $G$. Then we claim (1) that $\mathcal{L C}_{I_{0}}(G)$ is not left-recursive, and (2) that for all $L \subset L_{0}, \mathcal{L C}_{L}(G)$ is left-recursive.

Claim 1 follows from the fact that if $A \Rightarrow L_{L_{0}} B \gamma$ then $A \Rightarrow P B \gamma$ and the constraints in section 2.3 on useful productions of $\mathcal{L C}_{I_{0}}(G)$. Claim 2 follows from the fact that if $L \subset L_{0}$ then there is a chain of left-recursive productions that includes a top-down production; a simple induction on the length of the chain shows that $\mathcal{L C}_{L}(G)$ is left-recursive.

This result justifies the common practice in natural language left-corner parsing of taking the terminals to be the preterminal part-of-speech tags, rather than the lexical items themselves. (Wo did not attempt to calculate the size of such a left-conner grammar in the empirical cvaluation below, but it would be much larger than any of the grammars described there). In fact, if the preterminals are distinct from the other nonterminals (as they are in the tree-bank grammars investigated below) then $L_{0}$ does not include any productions beginning with a preterminal, and $\mathcal{L C}_{L_{0}}(G)$ contains no instances of schema la at all. We now turn our attention to the other schemata of the solcctive left-corner grammar transform.

\subsection{Factoring the output of $\mathcal{L} C_{L}$}

This section defines two factorizations of the output of the selective left-corner grammar transform that can dramatically reduce its size. These factorizations are most effective if the number of productions is much larger than the number of nonterminals, as is usually the case with tree-bank grammars.

The top-down factorization decomposes schema $1 b$ by introducing new nonterminals $D^{\prime}$, where $D \in V$, that have the same expansions that $D$ does in $G$. Using the same interpretation for variables as in schemata 1 , if $G=(V, T, P, S)$ then $\mathcal{L C}_{L}^{(t d)}(G)=\left(V_{t d}, T, P_{t d}, S\right)$, where:

$$
V_{t d}=V_{1} \cup\left\{D^{\prime}: D \in V\right\}
$$

and $P_{t d}$ contains all instances of the schemata 1a,
$3 \mathrm{a}, 3 \mathrm{~b}, 1 \mathrm{c}$ and $1 \mathrm{~d}$.

$$
\begin{array}{ll}
D \rightarrow A^{\prime} D-A & \text { where } A \rightarrow \alpha \in P-L \\
A^{\prime} \rightarrow \alpha & \text { where } A \rightarrow \alpha \in P-L
\end{array}
$$

Notice that the number of instances of schema $3 \mathrm{a}$ is less than the square of the number of nonterminals and that the number of instances of schema $3 \mathrm{~b}$ is the number of top-down productions; the sum of these numbers is usually much less than the number of instances of schema $1 \mathrm{~b}$.

Top-down factoring plays approximately the same role as "non-left-recursion grouping" (NLRG) does in Moore's (2000) approach. The major difference is that NLRG applies to all productions $A \rightarrow B \beta$ in which $B$ is not left-recursive, i.e., $\nexists \nexists \gamma . B \Rightarrow+, B \gamma$, while in our system top-down factorization applies to those productions for which $\nexists \gamma . B \Rightarrow_{P}^{\star} A \gamma$, i.e., the productions not directly involved in left recursion.

The left-corner factorization decomposes schema $1 c$ in a similar way using new nonterminals $D \backslash X$, where $D \in V$ and $X \in V \cup T$. $\mathcal{L C} \mathcal{C}_{L}^{(l c)}(G)=\left(V_{l c}, T, P_{l c}, S\right)$, where:

$$
V_{l c}=V_{1} \cup\{D \backslash X: D \in V, X \in V \cup T\}
$$

and $P_{l e}$ contains all instances of the schemata 1 a, $1 \mathrm{~b}, 4 \mathrm{a}, 4 \mathrm{~b}$ and $1 \mathrm{~d}$.

$$
\begin{array}{lll}
D-B \rightarrow C \backslash B D-C & \text { where } C \rightarrow B \beta \in L & \text { (4a) } \\
C \backslash B \rightarrow \beta & \text { where } C \rightarrow B \beta \in L & \text { (4b) }
\end{array}
$$

The number of instances of schema $4 a$ is bounded by the number of instances of schema $1 c$ and is typically much smaller, while the number of instances of schema $4 \mathrm{~b}$ is precisely the number of left-comer productions $L$.

Left-cornor factoring sems to correspond to one step of Moore's (2000) "left factor" (LF) opcration. The left factor operation constructs new nonterminals corresponding to common prefixes of arbitiary length, while left-corner factoring effectively only factors the first nonterminal symbol on the right hand side of left-corner productions. While we have not done experiments, Moore's left factor operation would seem to reduce the total number of symbols in the transformed grammar at the expense of possibly introducing additional productions, while our left-corner factoring reduces the number of productions.

These two factorizations can be used together in the obvious way to define a grammar transform $\mathcal{L C}_{I}^{(t d, l c)}$, whose productions are defined by schemata $1 \mathrm{a}, 3 \mathrm{a}, 3 \mathrm{~b}, 4 \mathrm{a}, 4 \mathrm{~b}$ and $1 \mathrm{~d}$. There are corresponding tree transforms, which we refer to as $\mathcal{T}_{I}^{(t d)}$, etc., below. Of course, the pruning constraints described in section 2.3 are applicable with these factorizations, and corresponding invertible trec transforms can be constructed. 


\section{Empirical Results}

To examine the effect of the transforms outlined above, we experimented with various PCFGs induced from sections $2-21$ of a modificd Pem WS.J trec-bank as described in Johnson (1998b) (i.c., labels simplificd to grammatical catcgorics, noot nodes added, ompty nodes and vacuous unary branches deleted, and auxiliarios retagged as $A U X$ or $\Lambda U X(1)$. We ignored lexical itons, and treated the part-of-speoch tiags as tominals. As Bob Moore? pointed out to us, the left-corner transform may produce left-recursive grammars if its input grammar contains unary cycles, so wo renoved them using the a transform that Moore suggested. Given an initial set of (non-epsilon) productions $P$, the transformed grammar contains the following productions, where the $A^{\text {th }}$ aro new non-torninals:

$$
\begin{array}{ll}
A \rightarrow \alpha & \text { where } A \rightarrow \alpha \in P, A \not P_{I}^{+} A \\
A \rightarrow D^{\natural} & \text { where } A \Rightarrow{ }_{P}^{*} D \Rightarrow+A_{I}^{+} \\
A^{\natural} \rightarrow \alpha & \text { where } A \rightarrow \alpha \in I^{+}, A \Rightarrow_{I}^{+}, A, \alpha \Rightarrow_{1}^{*}, A
\end{array}
$$

This transform can be extended to one on PCFGs which preserves derivation probeblilities. In this section, wo fix $P$ to be the productions that josult after applying this unary cycle semoval transformation to the trec-bank productions, and $G$ to be tho corresponding grammar.

'Lables 1 and 2 give the sizes of selective leftcornor grammar transforms of $G$ for various values of the left-corner set $L$ and factorizations, without and with epsilon-moval respectively. In the tables, $I_{20}$ is the set of left-recmesive productions in $I^{\prime}$, ats defined in section 2.4. $N$ is the set of productions in l' whose left-hand sicles do not, begin with a part-of-spech (POS) tag; because l'OS tags are distinct from other nonterminals in the tree-bank, $N$ is an easily identified set of productions guaranteed to include $L_{0}$. Tho tables also gives the sizes of maximum-likelihood PCFGs estimated from the trecs resulting from applying the selective left-coner trec transforms $\mathcal{T}$ to the trec-bank, breaking mary cycles as described above. For the parsing experiments bolow we always deleted empty nodes in the output of these tree transforns; this corresponds to epsilon removal in the grammar transform.

First, note that $\mathcal{L C} C_{1}(G)$, the result of applying the standard left-cornor grammar transform to $G$, has approximately 20 times the number of productions that $G$ has. However $\mathcal{L C}_{L_{0}}^{(t d, l c)}(G)$, the result of applying the solective left-corner grammar transformation with factorization, has ajproximatcly 1.4 times the number of productions that $G$ has. Thus the unethods described in this paper can in fact dramatically reduce the size of left-comer transformed grammars. Second, note that $\mathcal{L} \mathcal{C}_{N}^{(t d, l c)}(G)$ is not much larger than $\mathcal{L C C}_{L_{0}}^{(l d, l c)}(G)$. This is because $N$ is not

\begin{tabular}{c|rrrr} 
& \multicolumn{1}{|c}{ none } & $(t d)$ & $(l c)$ & $(t d, l c)$ \\
\hline$G$ & 15,040 & & & \\
$\mathcal{L} C_{l}$, & 346,344 & & 30,716 & \\
$\mathcal{L} C_{N}$ & 345,272 & 113,616 & 254,067 & 22,411 \\
$\mathcal{L} C_{L 0}$ & 314,555 & 103,504 & 232,415 & 21,364 \\
$\mathcal{T}_{I}$ & 20,087 & & 17,146 & \\
$\mathcal{T}_{N}$ & 19,619 & 16,349 & 19,002 & 15,732 \\
$\mathcal{T}_{l_{0}}$ & 18,945 & 16,126 & 18,437 & 15,618
\end{tabular}

Table 1: Sizes of PCFGs inferred using various grammar and tree transforms after pruning with link constraints without epsilon removal. Columns indicate factorization. In the grammar and tree transforms, $P$ is the set of productions in $G$ (i.e. the standard left-corner transform), $N$ is the set of all productions in $P$ which do not begin with a POS tag, and $L_{0}$ is the set of left-1ecursive productions.

\begin{tabular}{c|ccrc} 
& nonc & $(t d)$ & \multicolumn{1}{c}{$(l c)$} & $(t d, l c)$ \\
\hline $\mathcal{L} C_{l^{\prime}}$ & 564,430 & & 38,489 & \\
$\mathcal{L C} C_{N}$ & 563,295 & 176,644 & 411,986 & 25,335 \\
$\mathcal{L} C_{L_{0}}$ & 505,435 & 157,899 & 371,102 & 23,506 \\
$\mathcal{T}_{l}$ & 22,035 & & 17,398 & \\
$\mathcal{T}_{N}$ & 21,589 & 16,688 & 20,696 & 15,795 \\
$\mathcal{T}_{L_{0}}$ & 21,061 & 16,566 & 20,168 & 15,673
\end{tabular}

Table 2: Sizes of PCFGs inferred using various grammas and tree transforms after pruning with link constraints with epsilon removal, using the sane notation as lable 1.

much larger than $I_{0}$, which in turn is because most pairs of non-P'OS nonterminals $A, B$ are mutnally left-uecursive.

Tuming now to the PCFGs estimated after applying tree transforms, we notice that grammar sizo does not incroase noarly so dramatically. These PCFGs cncode a maximum-likelihood estimate of the state transition probabilities for various stochastic gencralized left-corner parsers, since a top-kown parser using these grammars simulates a gencralized left-corner parser. The fact that $\mathcal{L C}_{l},(G)$ is 17 times larger than the PCFG inferred after applying $\mathcal{T}_{p}$, to the trec-bank means that most of the possible transitions of a standard stochastic left-comer parser are not observed in the trec-bank training datal. The state of a left-coner parser does capture some lingnistic gencralizations (Manning and Carpenter, 1997; Roark and Jolnson, 1999), but one might still expect sparse-data problems. Note that $\mathcal{L C} \mathcal{C}_{L_{0}}^{(t, l, l c)}$ is only 1.4 times larger than $\mathcal{T}_{L_{0}}^{(t d, l c)}$, so we expect less sorious sparse data problens with the factored solective left-comer transform.

Wo quantify these sparse data problems in two ways using a held-out test corpus, viz., all sentences in section 23 of the trec-bank. First, table 3 lists the number of sentences in the test corpus that fail to receive a parse with the various PCFGs mentioned 


\begin{tabular}{c|cccc} 
Transform & none & $(t d)$ & $(l c)$ & $(t d, l c)$ \\
\hline nonc & 0 & & & \\
$\mathcal{T}_{P}$ & 2 & & 0 & \\
$\mathcal{T}_{N}$ & 2 & 0 & 2 & 0 \\
$\mathcal{T}_{L_{0}}$ & 0 & 0 & 0 & 0
\end{tabular}

Table 3: The number of sentences in section 23 that do not receive a parse using various grammars estimated from sections 2-21.

\begin{tabular}{c|cccc} 
Transform & none & $(t d)$ & $(l c)$ & $(t d, l c)$ \\
\hline none & 514 & & & \\
$\mathcal{T}_{P}$ & 665 & & 535 & \\
$\mathcal{T}_{N}$ & 664 & 543 & 639 & 518 \\
$\mathcal{T}_{L_{0}}$ & 640 & 547 & 615 & 522 \\
$\mathcal{T}_{P \epsilon}$ & 719 & & 539 & \\
$\mathcal{T}_{N_{\epsilon}}$ & 718 & 554 & 685 & 521 \\
$\mathcal{T}_{L_{0} \epsilon}$ & 706 & 561 & 666 & 521
\end{tabular}

Table 4: The number of productions found in the transformed trees of sentences in section 23 that do not appear in the corresponding transformed trees from sections 2 21. (The subscript epsilon indicates epsilon removal was applied).

above. This is a relatively crude measure, but correlates roughly with the ratios of grammar sizes, as expected.

Sccond, table 4 lists the number of productions found in the trec-transformed test corpus that do not appear in the correspondingly transformed trees of sections $2-21$. What is striking here is that the number of missing productions after either of the transforms $\mathcal{T}_{I_{0}}^{(t d, l c)}$ or $\mathcal{T}_{N}^{(t, d, l c)}$ is approximately the same as the number of missing productions using the untransformed trees, indicating that the factored solective left-corner transforms cause little or no additional sparse data problem. (The relationship between local trees in the parse trees of $G$ and $\mathcal{L C}_{L}(G)$ mentioned earlier implies that left-corner tree transformations will not decrease the number of missing productions).

We also investigate the accuracy of the maximumlikelihood parses (MLPs) obtained using the PCFGs estimated from the output of the various left-corner tree transforms. ${ }^{1}$ We searched for these parses using an exhraustive CKY parser. Because the parse trees of these PCFGs are isomorphic to the derivations of the corresponding stochastic gencralized left-corner parsers, we are in fact cvaluating different kinds of stochastic generalized left-corner parsers inferred from sections $2-21$ of the tree-bank. We used

\footnotetext{
${ }^{1}$ We did not investigate the grammars produced by the various left-corner grammar transforms. Because a left-corner grammar transform $\mathcal{L C}_{I}$. preserves production probabilities, the highest; scoring parses obtained using the woighted CFG $\mathcal{L C}_{L}(G)$ should be the highest scoring parses obtained using $G$ transformed by $\mathcal{T}_{L}$.
}

\begin{tabular}{c|cccc} 
& none & $(t d)$ & $(l c)$ & $(t d, l c)$ \\
\hline none & $70.8,75.3$ & & & \\
$\mathcal{T}_{I}$ & $75.8,77.7$ & & $74.8,76.9$ & \\
$\mathcal{T}_{N}$ & $75.8,77.6$ & $73.8,75.8$ & $75.5,77.8$ & $72.8,75.4$ \\
$\mathcal{T}_{J_{0}}$ & $75.8,77.4$ & $73.0,74.7$ & $75.6,77.8$ & $72.9,75.4$
\end{tabular}

Table 5: Labelled recall and precision scores of PCFGs estimated using various trec-transforms in a transformdetransform framework using test data from section 23 .

the transform-detransform framcwork described in Johnson (1998b) to evaluate the parses, i.e., we applied the appropriate inverse tree transform $\mathcal{T}^{-1}$ to detransform the parse trees produced using the PCFG estimated from trees transformed by $\mathcal{T}$. By calculating the labelled precision and recall scorcs for the detransformed trees in the usual manner, we can systematically compare the parsing accuracy of different kinds of stochastic generalized left-corner parsers.

Table 5 presents the results of this comparison. As reported previously, the standard left-comer grammar cmbeds sufficient non-local information in its productions to significantly improve the labelled precision and recall of its MLP's with respect to MLPs of the PCFG estimated from the untransformed trees (Manning and Carpenter, 1997; Roark and Johnson, 1999). Parsing accuracy drops off as grammar size decreases, presumably because smaller PCFGs have fewer adjustahle parameters with which to doscribe this non-local information. There are other kinds of non-local information which can be incorporated into a PCFG using a transform-detransform approach that result in an even greater improvennent of parsing accuracy (Johnson, 1998b). Ultimately, however, it scems that a more complex approach incorporating back-off and smoothing is necessary in order to achieve the parsing accuracy achieved by Charniak (1997) and Collins (1997).

\section{Conclusion}

This paper presented factored selective left-corner grammar transforms. These transforms preserve the primary benefits of the left-corner grammar transform (i.c., climination of left-recursion and preservation of annotations on productions) while dramatically ameliorating its principal problems (grammar size and sparse data problems). This should extend the applicability of left-corner techniques to situations involving large grammars. We showed how to identify the minimal set $L_{0}$ of productions of a grammar that must be recognized left-corner in order for the transformed grammar not to be left-recursive. We also proposed two factorizations of the output of the sclective left-corner grammar transform which further reduce grammar size, and showed that there is only a minor increase in grammar size when the 
factored selective left-comor transform is applied to a large tres-bank grammar. Finally, we exploited the tree transforms that correspond to these grammar transforms to formulate and study a class of stochastic generalized left-corner parsers.

This work could be extended in a number of ways. For example, in this paper we assumed that one would always choose a left-comer production set that includes the minimal set $L_{0}$ recuired to cnsure that the transformed grammar is not left-recursive. However, Roark and Johnson (1999) report good performance from a stochastically-guided top-down parser, suggesting that left-recursion is not always fatal. It might be possible to judiciously choose a left-corner production set smaller than $L_{0}$ which eliminates pernicious left-recursion, so that the romaining left-recursive cycles have such low probability that they will effectively never loe used and a stochastically-guided top-down parser will nover scarch them.

\section{References}

Stephen Abney and Mark Johnson. 1991. Monory requirements and local ambiguities of parsing strategies. Journal of P'sycholinguistic Rescarch, 20(3):233 -250.

Steven Abney, David McAllester, and Fernando Pereira. 1999. Relating probabilistic grammars and antomata. In Procectings of the 37 th Annual Mecting of the Association for Computational Linguistics, pages 542-549, San Francisco. Morgan Kaufmann.

Alfred V. Aho and Jeffory D. Ullman. 1972. The Theory of Parsing, Translation and Compiting; Volume: 1 : Parsing. P'rentice-Hall, Englewood Cliffs, New Jersey.

Engene Clamiak. 1997. Statistical parsing with a context-freo grammar and word statistics. In Procedings of the Fourteenth National Conference on Artificial Intclligence, Monlo Park. AAAI Press/MIT P'ress.

Michacl Collins. 1997. Three generative, lexicalised models for statistical parsing. In The Procecdings of the 35th Annual Mecting of the Association for Computational Linguistics, San Francisco. Morgan Kaufmannn.

Michacl A. Covington. 1994. Natural Language I'rocessing for Prolog Programmers. Prentice Irall, Englewood Clifls, New Jersey.

A. Demers. 1977. Generalized left-corner parsing. In Confercnec Record of the Fourth ACM Symposium on Principles of Programming languages, 1977 ACM SIGACT/SIGPLAN, pages 170 - 182 .

John E. Hopcroft and Jeffrey D. Ullmanl. 1979. Introduction to Automata Theory, Languages and Computation. Addison-Wesley.

Mark Johnson. 1998a. Finite state approximation of unification grammars using left-corner grammar transforms. In The Proceclirgs of the $30 \mathrm{hh}$ Annual Conference of the Association for Computational Iinguistics (COLING-ACl), pages 619-623. Morgan Kalufinamn.

Mark Johnson. 1998b. PCTG models of linguis- tic: tre representations. Computational Linguistics, $24(4): 613-632$.

Christopher D. Manning and Bob Carpenter. 1997. Probabilistic parsing using left-corner models. In Procecdings of the 5th International Workshop on Parsing Teclunologies, pages 147-158, Massachusetts Institute of Technology.

Yuji Matsumoto, Hozumi Tanaka, Hideki Hirakawa, IIideo Miyoshi, and Hideli Yasukawa. 1983. BUP: A bottom-up parser embedded in Prolog. New Gencration Computing, 1(2):145-158.

liobert C. Moorc. 2000. Removing left recursion from context-free grammars. In Proceedings of 1st Annual Conference of the North American Chapter of the Association for Computational Linguistics, San Francisco. Morgan Kaufmann.

Anton Nijholt. 1980. Context-free Grammars: Covers, Normal Forms, and Parsing. Springer Vorlag, Borlin.

Fermando C.N. Percira and Stuart M. Shicher. 1987. Prolog and Natural Language Analysis. Number 10 in CSLI Lecture Notes Series. Chicago University Press, Chicago.

Philip Resnik. 3992. Jeft-corner parsing and psychological plausibility. In The Procecdings of the fiftecnth Intemational Conference on Computational Linguistics, COLING-92, volume 1, pages 191-197.

J3rian Roark and Mark Johnson. 1999. Efficient probabilistic: top-down and left-corner parsing. In I'rocecdings of the 37th Annual Mecting of the ACL, pages $421-428$.

Stanley J. Rosenkrantz and Philip M. Lewis II. 1970. Deterministic: left corner parser. In IEEE Conferenece Record of the 11th Annual Symposium on Suitching and Automata, pages $139 \cdot 152$.

Gertjan van Noort. 1997. An efficient implementation of the heal-corner parser. Computational linguistics, $23(3): 425-456$. 